See discussions, stats, and author profiles for this publication at: https://www.researchgate.net/publication/331872507

\title{
Space-Time Media-Based Modulation
}

Article in IEEE Transactions on Signal Processing · March 2019

DOI: 10.1109/TSP.2019.2905836

CITATIONS

2

2 authors:

Zehra Yigit

Istanbul Technical University

7 PUBLICATIONS 37 CITATIONS

SEE PROFILE
READS

188

Some of the authors of this publication are also working on these related projects:

CALL FOR PAPERS: "Chaotic and Index Modulations: Emerging Paradigms for Future Wireless Communications" View project

Code Index Modulation and Spatial Modulation: A New High Rate and Energy Efficient Scheme for MIMO systems View project 


\title{
Space-Time Media-Based Modulation
}

\author{
Zehra Yigit, Student Member, IEEE and Ertugrul Basar, Senior Member, IEEE
}

\begin{abstract}
Media-based modulation (MBM), which utilizes radiation patterns of a reconfigurable antenna to convey information, appears as a promising index modulation (IM) scheme for beyond 5G networking. In this paper, we present a general framework for MBM from the perspective of space-time coding, and introduce a novel space-time coded IM concept, which is called space-time media-based modulation (ST-MBM). The proposed scheme is based on one of the prominent IM solutions, space shift keying (SSK), along with Hurwitz-Radon family of matrices in order to achieve transmit diversity gain with a single radio frequency (RF) chain by utilizing the unique $R F$ mirror activation principle of MBM. We derive the theoretical pairwise error probability of the ST-MBM scheme for correlated and uncorrelated channel states and obtain the average bit error probability (ABEP). Additionally, a lower bound is derived for the mutual information of the ST-MBM scheme to gain insights into the information theoretical bounds of the proposed scheme. Furthermore, extensive computer simulations are provided to show the superior error performance of the ST-MBM scheme over the state-of-the-art multiple-input multiple-output (MIMO)based transmission systems.
\end{abstract}

Index Terms-Space shift keying (SSK), media-based modulation (MBM), index modulation (IM), MIMO systems, space-time coding.

\section{INTRODUCTION}

$\mathbf{T}$ HE evolution of multiple-input multiple-output (MIMO) technology has boosted the overall performance of traditional communication systems from many different aspects. Capability of the MIMO systems to meet the growing demand on higher data rates and higher capacity significantly accelerates the development of today's wireless technologies. Consequently, MIMO techniques have been widely used in wireless standards including Long Term Evolution (LTE), IEEE 802.11x (Wi-Fi) and IEEE 802.16 (WiMAX). According to the conventional MIMO transmission concept [1], since all transmit antennas are used for signaling, an increasing number of transmit antennas leads to a higher data rate and, at the same time, remarkably increases the transceiver complexity, which is considered as the main drawback of these early designs.

Over the past decade, there has been a growing trend towards increasing the data rate by conveying additional information bits through the building blocks of a MIMO transmission system by the novel concept of index modulation (IM) [2]. Compared to the early designs [1], with their considerably lower transceiver complexity and higher energy efficiency, IM schemes have been considered as alternative solutions for $5 \mathrm{G}$ and beyond wireless networks [2].

Z. Yigit is with the Department of Electronics and Communication Engineering, Istanbul Technical University, Maslak 34469, Istanbul, Turkey. email: yigitz@itu.edu.tr

E. Basar is with the Communications Research and Innovation Laboratory (CoreLab), Department of Electrical and Electronics Engineering, Koç University, Sariyer 34450, Istanbul, Turkey. e-mail: ebasar@ku.edu.tr
Spatial modulation (SM) [3], which utilizes the indices of the available transmit antennas of a MIMO system to convey extra information bits besides the conventional modulation bits, has been regarded as the pioneer of IM techniques. Later, in [4], space shift keying (SSK) scheme, which only transmits information bits by specifying index of the active transmit antenna is presented. Over the past few years, numerous follow-up studies on SM have been performed under diverse research fields [5]. Furthermore, the concept of IM has found many application areas in multi-carrier communications [6], [7], spread spectrum communication systems [8], [9], optical wireless communications [10], [11], and so on.

Another novel IM transmission technique called mediabased modulation (MBM), which uses radio frequency (RF) mirrors in an innovative way to convey data bits, is introduced in [12]. In the MBM scheme, each transmit antenna is equipped with RF mirrors where ON/OFF status of these RF mirrors create different channel fade realizations from the perspective of the receiver. Despite the fact that it is a relatively new concept, there is an increasing interest in the concept of MBM over the past two years. Traditional SM [3] and quadrature SM (QSM) [13] systems are adapted to the MBM concept in [14] and [15], respectively. More recently, the works of [16] and [17] integrated MBM with Alamouti's space-time block code (STBC) [18] and uncoded space-time labeling diversity (USTLD) [19], respectively. Also, MBMbased multi-user networks [20] and uplink massive MBMMIMO systems [21], [22] are proposed. To decrease the receiver complexity, iterative interference cancellation [23], constrained linear programming [24] and message passing based [25] suboptimal detectors are designed, and the performance of the MBM scheme is investigated in the presence of imperfect channel estimation [26]. Very recently, MBMbased full-duplex [27] and secrecy communication [28], [29] systems are developed. Interested readers are refered to [30] for a recent tutorial on MBM technologies.

Both in SSK and in the simplest form of MBM, which does not convey any information with an ordinary modulation, the received signal is perceived as a random variable with complex Gaussian distribution, which is the desired way to achieve the maximum capacity [31]. Unlike conventional MIMO systems, this phenomenon plays an important role to understand the reason behind the improved error performance of SSK and MBM schemes with increasing number of transmit antennas [16]. The method used in SSK and MBM to perceive a complex Gaussian signal at the receiver is the multiplication of the channel matrix $\mathbf{H}$ with a sparse transmission vector that includes only one non-zero element. Alternatively, conveying a matrix, whose each row or column corresponding to an SSK vector, is another way to perceive complex Gaussian distributed random variables at the receiver, which can be 


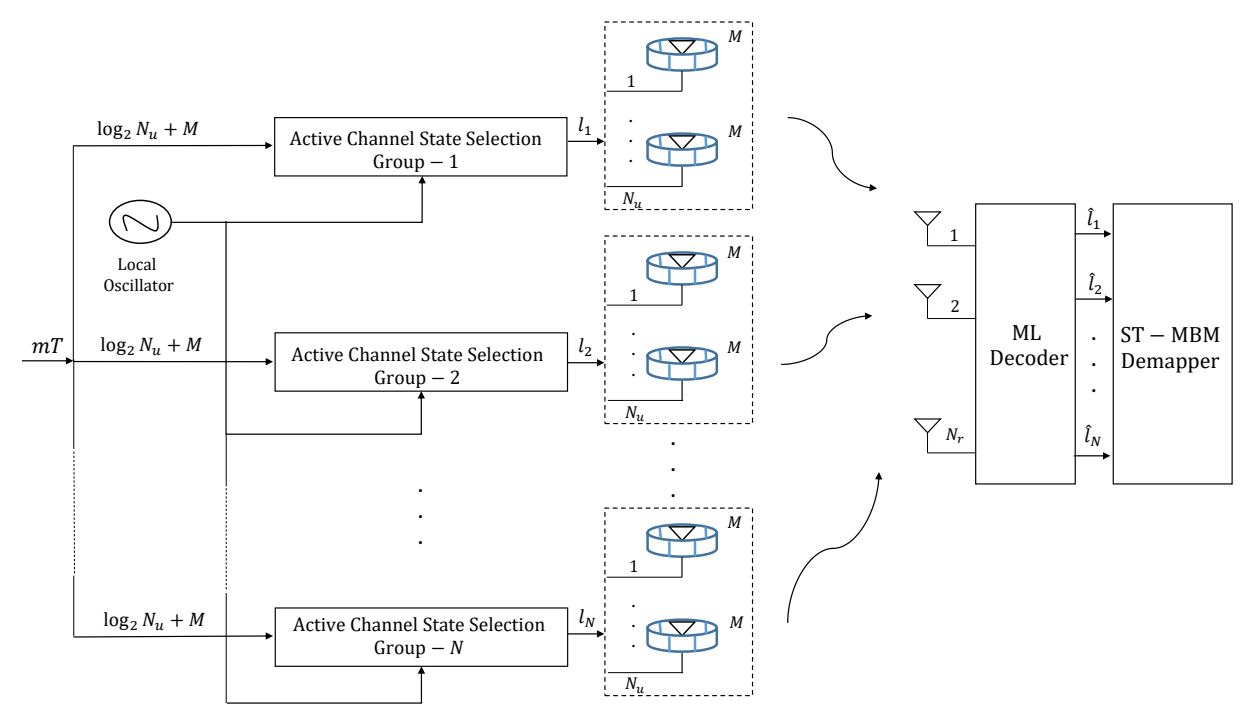

Fig. 1. Block diagram of the ST-MBM scheme.

carried out with the idea of spreading the transmitted signals in time by considering space-time coding principle. Furthermore, space-time designs can be exploited to achieve transmit diversity for MBM systems with a considerably lower transceiver complexity.

In this paper, an innovative concept based on the framework of SSK and MBM schemes, called space-time media-based modulation (ST-MBM), is proposed by cleverly combining the Hurwitz-Radon family of matrices [32] with the MBM transmission approach. The proposed ST-MBM scheme is the first STBC-based scheme that achieves transmit diversity gains by using a single RF chain with a significantly lower receiver complexity. Theoretical error performance analysis of the proposed ST-MBM scheme is performed and its exact average bit error probability (ABEP) is derived for correlated and uncorrelated channel states. Furthermore, a lower bound is obtained for the mutual information of the ST-MBM scheme. Through comprehensive computer simulations, bit error rate (BER) performance of ST-MBM scheme is compared with the existing state-of-the-art MIMO concepts in the literature.

The remaining of the paper is organized as follows. The system model of the proposed ST-MBM scheme is introduced in Section II. In Section III, theoretical error performance and capacity analyses of the ST-MBM scheme are performed. Computer simulation results are presented in Section IV and the paper is concluded in Section V.

Notation: Bold capital and lowercase letters are used for matrices and vectors, respectively. Transposition and Hermitian transposition operators are denoted by $(\cdot)^{T}$ and $(\cdot)^{H}$, respectively. $\operatorname{Tr}(\cdot), \operatorname{rank}(\cdot)$ and $\operatorname{det}(\cdot)$ respectively stand for the trace, the rank and the determinant of a matrix. $\|\cdot\|$ stands for the Euclidean/Frobenius norm and $\operatorname{vec}(\cdot)$ represents the vectorization operator. The complex Gaussian distribution of a random variable $x$ with $m$ mean and $\sigma^{2}$ variance is denoted by $\mathcal{C N}\left(m, \sigma^{2}\right) \cdot \operatorname{Pr}(\cdot)$ stands for the probability of an event and $\mathbb{E}(\cdot)$ denotes expectation. $Q$-function, the entropy function and the Kronecker product are represented by $Q(\cdot), H(\cdot)$ and $\otimes$, respectively. $\mathbf{I}_{m}$ denotes the $m \times m$ identity matrix and $\mathbb{C}^{m \times n}$ represents the set of matrices with dimensions of $m \times n$.

\section{Space-Time Media Based Modulation}

An efficient way to compensate the inherently low spectral efficiency of STBC-based systems is to carry as much information as possible via the indices of the building blocks of the target transmission system. For a MIMO-MBM transmission scheme, the available building blocks for indexing are transmit antennas and RF mirrors. Beside these, in the proposed STMBM scheme, in order to further improve the spectral efficiency, information bits are subdivided into $N$ transmission groups and space-time coding principle is independently applied to these transmission groups.

In Fig. 1, block diagram of the proposed ST-MBM scheme, which achieves transmit diversity by expanding the transmission to $T$ time slots while considering a MIMO configuration with $N_{t}$ transmit and $N_{r}$ receive antennas, is shown. In the STMBM scheme, each transmit antenna is surrounded by $M$ RF mirrors, where ON/OFF status of these $M$ RF mirrors generate $2^{M}$ different channel fade realizations for each of $N_{t}$ transmit antennas. As shown in Fig. 1, incoming $m T$ information bits and, at the same time, $N_{t}$ transmit antennas are subdivided into $N$ groups. In each of these transmission groups,

$$
\frac{m T}{N}=\log _{2}\left(N_{u}\right)+M
$$

bits are transmitted, where $N_{u}=N_{t} / N$ is defined as the number of transmit antennas in the $u$ th transmission group, for $u \in\{1,2, \ldots, N\}$. In each group, the first $\log _{2} N_{u}$ bits of the incoming $\log _{2}\left(N_{u}\right)+M$ bits determine the index of the active transmit antenna, which is selected out of $N_{u}$ available transmit antennas, while the following $M$ bits specify one of the available $2^{M}$ channel states corresponding to this active antenna. It can be alternatively expressed that in each transmission group, one of the available $P_{u}=N_{u} 2^{M}$ channel fade realizations, which are created jointly by reconfigurable antennas and the SSK concept, is selected by the incoming 
$\log _{2}\left(P_{u}\right)$ bits. Therefore, the total number of channel fade realizations through $N$ transmission groups becomes $P=N P_{u}$, and the spectral efficiency of the ST-MBM scheme in bits per channel use (bpcu) is given as

$$
m=\frac{N\left[M+\log _{2}\left(N_{u}\right)\right]}{T}=\frac{N \log _{2}\left(P_{u}\right)}{T}
$$

due to the use of $T$ time slot. The signaling structure of the proposed scheme in $T$ time slots will be explained next.

For each transmission group, the incoming bits determine the transmission vector of the first time slot. This corresponds to an SSK vector, since no information is conveyed through the selected channel state by means of amplitude/phase modulations. Therefore, the transmission vector of the first time slot related to transmission group $u$ can be given as

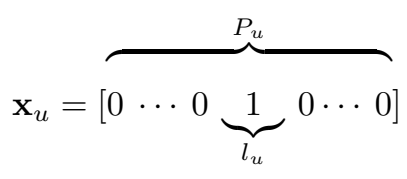

where $l_{u}$ denotes the index of the specified channel fade realization of the first time slot among $P_{u}$ channel fade realizations in the $u$ th transmission group, and $l_{u} \in\left\{1,2, \ldots, P_{u}\right\}$. Then, the overall transmission matrix of each group is formed by the following structure of the Hurwitz-Radon family of matrices, where a detailed discussion is given below.

At the receiver side, in order to perceive complex Gaussian distributed random variables as in the case of SSK/MBM and, at the same time, to obtain transmit diversity gain, the HurwitzRadon family of matrices [32], a set of $L \times L$ real orthogonal matrices whose each row and column corresponds to an SSK vector, are used as core STBCs. For $l_{u} \in\{1,2, \ldots, L\}$, each set of these $L \times L$ Hurwitz-Radon matrices satisfies the following conditions:

$$
\begin{aligned}
& \mathbf{B}_{l_{u}}{ }^{T} \mathbf{B}_{l_{u}}=\mathbf{I}_{L} \quad l_{u}=2, \ldots, L \\
& \mathbf{B}_{l_{u}}{ }^{T}=-\mathbf{B}_{l_{u}} \quad l_{u}=2, \ldots, L \\
& \mathbf{B}_{l_{u}} \mathbf{B}_{l_{u^{\prime}}}=-\mathbf{B}_{l_{u}{ }^{\prime}} \mathbf{B}_{l_{u}} \quad 1 \leq l_{u}<l_{u}^{\prime} \leq L
\end{aligned}
$$

where $\mathbf{B}_{1}=\mathbf{I}_{L}$ and $L \in\{2,4,8\}$.

For $L=4$, the Hurwitz-Radon matrices satisfying (4) are given as [32]

$$
\begin{aligned}
\mathbf{B}_{1}= & {\left[\begin{array}{llll}
1 & 0 & 0 & 0 \\
0 & 1 & 0 & 0 \\
0 & 0 & 1 & 0 \\
0 & 0 & 0 & 1
\end{array}\right], \mathbf{B}_{2}=\left[\begin{array}{cccc}
0 & 1 & 0 & 0 \\
-1 & 0 & 0 & 0 \\
0 & 0 & 0 & -1 \\
0 & 0 & 1 & 0
\end{array}\right] } \\
\mathbf{B}_{3}= & {\left[\begin{array}{cccc}
0 & 0 & 1 & 0 \\
0 & 0 & 0 & 1 \\
-1 & 0 & 0 & 0 \\
0 & -1 & 0 & 0
\end{array}\right], \mathbf{B}_{4}=\left[\begin{array}{cccc}
0 & 0 & 0 & 1 \\
0 & 0 & -1 & 0 \\
0 & 1 & 0 & 0 \\
-1 & 0 & 0 & 0
\end{array}\right] . }
\end{aligned}
$$

Similarly, the following Hurwitz-Radon matrices are constructed for $L=8$ [32]:

$$
\begin{aligned}
& \mathbf{B}_{2}=\left[\begin{array}{cccccccc}
0 & 1 & 0 & 0 & 0 & 0 & 0 & 0 \\
-1 & 0 & 0 & 0 & 0 & 0 & 0 & 0 \\
0 & 0 & 0 & -1 & 0 & 0 & 0 & 0 \\
0 & 0 & 1 & 0 & 0 & 0 & 0 & 0 \\
0 & 0 & 0 & 0 & 0 & -1 & 0 & 0 \\
0 & 0 & 0 & 0 & 1 & 0 & 0 & 0 \\
0 & 0 & 0 & 0 & 0 & 0 & 0 & 1 \\
0 & 0 & 0 & 0 & 0 & 0 & -1 & 0
\end{array}\right], \mathbf{B}_{3}=\left[\begin{array}{cccccccc}
0 & 0 & 1 & 0 & 0 & 0 & 0 & 0 \\
0 & 0 & 0 & 1 & 0 & 0 & 0 & 0 \\
-1 & 0 & 0 & 0 & 0 & 0 & 0 & 0 \\
0 & -1 & 0 & 0 & 0 & 0 & 0 & 0 \\
0 & 0 & 0 & 0 & 0 & 0 & 1 & 0 \\
0 & 0 & 0 & 0 & 0 & 0 & 0 & 1 \\
0 & 0 & 0 & 0 & -1 & 0 & 0 & 0 \\
0 & 0 & 0 & 0 & 0 & -1 & 0 & 0
\end{array}\right] \\
& \mathbf{B}_{4}=\left[\begin{array}{cccccccc}
0 & 0 & 0 & 1 & 0 & 0 & 0 & 0 \\
0 & 0 & -1 & 0 & 0 & 0 & 0 & 0 \\
0 & 1 & 0 & 0 & 0 & 0 & 0 & 0 \\
-1 & 0 & 0 & 0 & 0 & 0 & 0 & 0 \\
0 & 0 & 0 & 0 & 0 & 0 & 0 & 1 \\
0 & 0 & 0 & 0 & 0 & 0 & -1 & 0 \\
0 & 0 & 0 & 0 & 0 & 1 & 0 & 0 \\
0 & 0 & 0 & 0 & -1 & 0 & 0 & 0
\end{array}\right], \mathbf{B}_{5}=\left[\begin{array}{cccccccc}
0 & 0 & 0 & 0 & 1 & 0 & 0 & 0 \\
0 & 0 & 0 & 0 & 0 & 1 & 0 & 0 \\
0 & 0 & 0 & 0 & 0 & 0 & -1 & 0 \\
0 & 0 & 0 & 0 & 0 & 0 & 0 & -1 \\
-1 & 0 & 0 & 0 & 0 & 0 & 0 & 0 \\
0 & -1 & 0 & 0 & 0 & 0 & 0 & 0 \\
0 & 0 & 1 & 0 & 0 & 0 & 0 & 0 \\
0 & 0 & 0 & 1 & 0 & 0 & 0 & 0
\end{array}\right] \\
& \mathbf{B}_{6}=\left[\begin{array}{cccccccc}
0 & 0 & 0 & 0 & 0 & 1 & 0 & 0 \\
0 & 0 & 0 & 0 & -1 & 0 & 0 & 0 \\
0 & 0 & 0 & 0 & 0 & 0 & 0 & -1 \\
0 & 0 & 0 & 0 & 0 & 0 & 1 & 0 \\
0 & 1 & 0 & 0 & 0 & 0 & 0 & 0 \\
-1 & 0 & 0 & 0 & 0 & 0 & 0 & 0 \\
0 & 0 & 0 & -1 & 0 & 0 & 0 & 0 \\
0 & 0 & 1 & 0 & 0 & 0 & 0 & 0
\end{array}\right], \mathbf{B}_{7}=\left[\begin{array}{cccccccc}
0 & 0 & 0 & 0 & 0 & 0 & 1 & 0 \\
0 & 0 & 0 & 0 & 0 & 0 & 0 & -1 \\
0 & 0 & 0 & 1 & 0 & 0 & 0 & 0 \\
0 & 0 & 0 & 0 & 0 & -1 & 0 & 0 \\
0 & 0 & -1 & 0 & 0 & 0 & 0 & 0 \\
0 & 0 & 0 & 1 & 0 & 0 & 0 & 0 \\
-1 & 0 & 0 & 0 & 0 & 0 & 0 & 0 \\
0 & 1 & 0 & 0 & 0 & 0 & 0 & 0
\end{array}\right] \\
& \mathbf{B}_{8}=\left[\begin{array}{cccccccc}
0 & 0 & 0 & 0 & 0 & 0 & 0 & 1 \\
0 & 0 & 0 & 0 & 0 & 0 & 1 & 0 \\
0 & 0 & 0 & 0 & 0 & 1 & 0 & 0 \\
0 & 0 & 0 & 0 & 1 & 0 & 0 & 0 \\
0 & 0 & 0 & -1 & 0 & 0 & 0 & 0 \\
0 & 0 & -1 & 0 & 0 & 0 & 0 & 0 \\
0 & -1 & 0 & 0 & 0 & 0 & 0 & 0 \\
-1 & 0 & 0 & 0 & 0 & 0 & 0 & 0
\end{array}\right]
\end{aligned}
$$

where $\mathbf{B}_{1}=\mathbf{I}_{8}$. It is worth noting that real-orthogonal STBCs are constructed by using the above Hurwitz-Radon matrices [32].

In the proposed ST-MBM scheme, after specifying the transmission vectors of the first time slot for each of $N$ transmission groups (3), the Hurwitz-Radon matrices are independently exploited for each group as the core STBCs to construct the overall transmission matrix. For $P_{u}=T=L$, the rows and columns of the Hurwitz-Radon matrices are considered for $T$ time slots and $P_{u}$ channel fade realizations, respectively.

Let us introduce the ST-MBM concept for $P_{u}=T$ with the following example, while the generalized ST-MBM concept for larger channel fade realizations of $P_{u}>8$ will be given in the next subsection.

Example: Assume that $P_{u}=4$ channel fade realizations are generated in each of $N=2$ transmission groups, where $N_{t}=4$ transmit antennas are equipped with a single $(M=1)$ RF mirror. In this setup, spreading the signal transmission takes place in $T=4$ time slots. For this case, a spectral efficiency of $m=1 \mathrm{bpcu}$ is achieved, where $N_{u}=N_{t} / N=2$ and $u \in\{1,2\}$. Suppose that incoming $m T=4$ bits of $\left\{\begin{array}{llll}1 & 0 & 0 & 1\end{array}\right\}$ are transmitted over $N=2$ transmission groups, where the first two $\left\{\begin{array}{ll}1 & 0\end{array}\right\}$ bits are assigned to the first group and the remaining $\left\{\begin{array}{lll}0 & 1\end{array}\right\}$ bits are assigned to the second group. In the first group, the first $\{1\}$ bit of $\{10\}$ bit sequence activates one of $N_{1}=2$ transmit antennas while the following 
$\{0\}$ bit selects one of $2^{M}=2$ channel states generated with $M=1 \mathrm{RF}$ mirror, which corresponds to the first channel state of the second transmit antenna. It can be alternatively stated that the bit sequence of $\{10\}$ specifies the third channel fade realization $\left(l_{1}=3\right)$ among $P_{1}=N_{1} 2^{M}=4$ channel fade realizations. Similarly, for the second group, the first $\{0\}$ bit of remaining $\left\{\begin{array}{ll}0 & 1\end{array}\right\}$ bits activates the first transmit antenna of the second antenna group, where $N_{2}=2$, while the following $\{1\}$ bit determines the second channel state of the corresponding active antenna. In other words, the second $\left(l_{2}=2\right)$ out of $P_{2}=N_{2} 2^{M}=4$ channel fade realizations is selected. For the remaining three time slots, we follow the HurwitzRadon matrices in (5) to obtain a diversity gain. Therefore, transmission matrices of the first and second groups can be given as

$$
\mathbf{X}_{1}=\left[\begin{array}{cccc}
0 & 0 & 1 & 0 \\
0 & 0 & 0 & 1 \\
-1 & 0 & 0 & 0 \\
0 & -1 & 0 & 0
\end{array}\right], \mathbf{X}_{2}=\left[\begin{array}{cccc}
0 & 1 & 0 & 0 \\
-1 & 0 & 0 & 0 \\
0 & 0 & 0 & -1 \\
0 & 0 & 1 & 0
\end{array}\right]
$$

which respectively correspond to $\mathbf{B}_{3}$ and $\mathbf{B}_{2}$ in (5). Then, the overall transmission matrix, that comprises both $\mathbf{X}_{1} \in \mathbb{C}^{T \times P_{1}}$ and $\mathbf{X}_{2} \in \mathbb{C}^{T \times P_{2}}$, is given as

$$
\mathbf{X}=\left[\begin{array}{cccc|cccc}
0 & 0 & 1 & 0 & 0 & 1 & 0 & 0 \\
0 & 0 & 0 & 1 & -1 & 0 & 0 & 0 \\
-1 & 0 & 0 & 0 & 0 & 0 & 0 & -1 \\
0 & -1 & 0 & 0 & 0 & 0 & 1 & 0
\end{array}\right]
$$

We note that the rows and columns of $\mathbf{X} \in \mathbb{C}^{T \times P}$ correspond to time slots and channel fade realizations, respectively. Since the overall transmission matrix is formed by the elements of $\{1,-1,0\}$ in the baseband, to transmit these elements, a single $\mathrm{RF}$ chain is sufficient. Then, as given in Fig. 1, a cosine carrier signal generated from a local oscillator is supplied to $N$ groups to transmit $1 \mathrm{~s}$ and -1 s. Thus, as the traditional SM/SSK systems [33], the overall ST-MBM system has been designed by using a single RF chain.

\section{A. Design Criteria and Transmit Diversity Analysis}

The rank and determinant criteria are commonly used in the design of STBCs to maximize diversity and coding gains. Let us consider the transmit STBC matrix $\mathbf{S}_{i}$ and the erroneously detected STBC matrix $\mathbf{S}_{j}$, for $i \neq j$, then transmit diversity gain $G_{d}$ is evaluated as

$$
G_{d}=\operatorname{rank}\left[\left(\mathbf{S}_{i}-\mathbf{S}_{j}\right)\left(\mathbf{S}_{i}-\mathbf{S}_{j}\right)^{H}\right] .
$$

The maximum transmit diversity order that can be achieved by any $T \times P_{u}$ orthogonal STBC is equal to the number of time slots $T$, which is respectively four and eight for the square STBC matrices given in (5) and (6). However, since there is only one non-zero element in each row and column of the Hurwitz-Radon matrices, removing any row(s) or column(s) results in a new orthogonal design, which does not violate the orthogonality of the remaining matrix and allows us to achieve any transmit diversity order of $G_{d}=T$ for $T \leq 8$. In the ST-MBM scheme, since the Hurwitz-Radon matrices are utilized as core STBC matrices, we take the advantage of this unique property and in the same way, can achieve a transmit diversity order of $T$, where $2 \leq T \leq 8$, by removing the required number of row(s) from the overall ST-MBM transmission matrix. Accordingly, in the ST-MBM scheme, when the transmit signal matrix $\mathbf{X}$ is erroneously detected as $\hat{\mathbf{X}}$, the transmit diversity order of the ST-MBM scheme is given as:

$$
G_{d}=\operatorname{rank}\left[(\mathbf{X}-\hat{\mathbf{X}})(\mathbf{X}-\hat{\mathbf{X}})^{H}\right]=T .
$$

Furthermore, considering the above rank criterion, inserting an all-zero matrix to any STBC matrix, without distorting the integrity of the target matrix, still does not violate the orthogonality of the core STBC, and the newly generated STBC matrix achieves the same transmit diversity as that of the core STBC. This allows us to generalize the STMBM scheme for more than eight channel fade realizations $\left(P_{u}>8\right)$, which exceeds the maximum dimensions of the Hurwitz-Radon matrix, and to attain higher spectral efficiency values while still achieving a transmit diversity order of $T \leq 8$. In each transmission group, since $P_{u}=N_{u} 2^{M}>8$ is also an integer multiple of 8 , to retain the orthogonality of the core STBC, the required number of $T \times 8$ all-zero matrices are added to the core $T \times 8$ STBC matrix, and the corresponding transmission matrix is generated by shifting the core STBC matrix in blocks in accordance with the index of active channel state $l_{u}$, where $l_{u} \in\left\{1,2, \ldots, P_{u}\right\}$. Therefore, for $P_{u}>8$, the overall transmission matrix of $u$ th transmission group, $\mathbf{X}_{u} \in \mathbb{C}^{T \times P_{u}}$, can be given as

$$
\mathbf{X}_{u}=\left[\begin{array}{lllllll}
\mathbf{0}_{8} & \cdots & \mathbf{0}_{8} & \mathbf{B}_{l_{u}} & \mathbf{0}_{8} & \cdots & \mathbf{0}_{8}
\end{array}\right]
$$

where $\mathbf{0}_{8}$ is a $T \times 8$ all-zero matrix. In (11), $\mathbf{B}_{l_{u}}$ either corresponds to one of $8 \times 8$ Hurwitz-Radon matrices that achieve the maximum transmit diversity for $T=8$, or it corresponds to a non-square STBC matrix obtained by extracting the required number of row(s) from the considered $8 \times 8$ Hurwitz-Radon matrix for $T<8$. Therefore, the overall STMBM transmission matrix $\mathbf{X} \in \mathbb{C}^{T \times P}$, for $P=N P_{u}$ being the number of all channel fade realizations that comprises of $N$ transmission groups, becomes

$$
\mathbf{X}=\left[\begin{array}{llll}
\mathbf{X}_{1} & \mathbf{X}_{2} & \cdots & \mathbf{X}_{N}
\end{array}\right]
$$

$\mathbf{X}$ is transmitted over a wireless channel, represented by $\mathbf{H} \in \mathbb{C}^{P \times N_{r}}$, while experiencing additive white Gaussian noise (AWGN), characterized by $\mathbf{W}$, whose elements are independent and identically distributed (i.i.d) complex Gaussian random variables with $\mathcal{C N}\left(0, N_{0}\right)$ distribution. Therefore, the matrix of received signals is given as

$$
\mathbf{Y}=\mathbf{X H}+\mathbf{W}
$$

where $\mathbf{Y} \in \mathbb{C}^{T \times N_{r}}$. Alternatively, the received signals can be represented by the following equivalent model as:

$$
\mathbf{y}=\mathbf{h}_{\chi}+\mathbf{w}=\sum_{u=1}^{N} \mathbf{h}_{u}+\mathbf{w}
$$




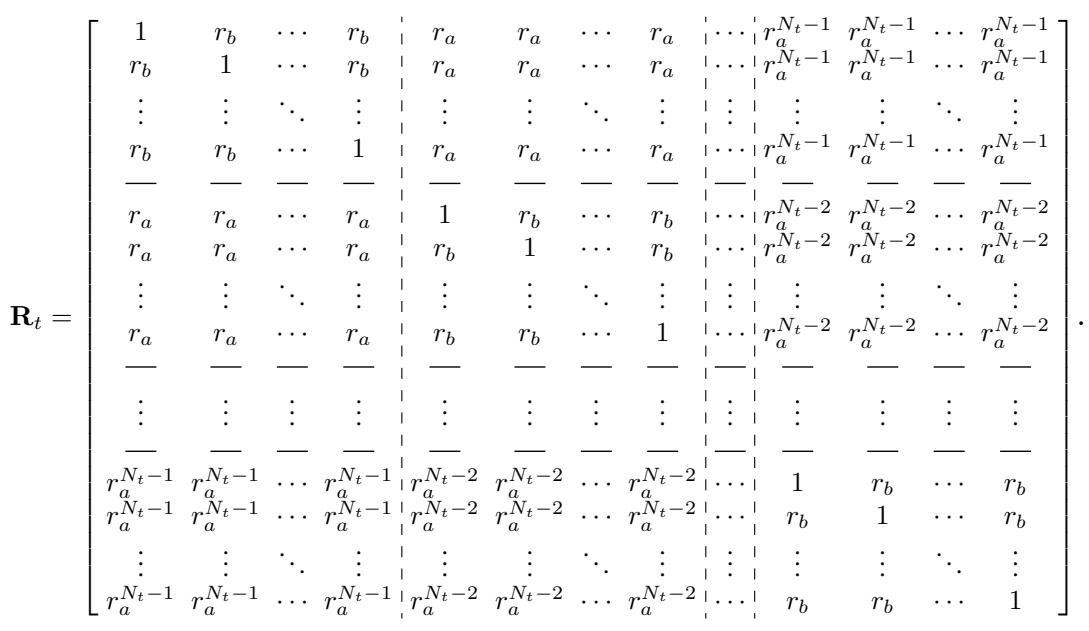

where $\mathbf{y} \in \mathbb{C}^{T N_{r} \times 1}=\operatorname{vec}(\mathbf{Y})$, w $\in \mathbb{C}^{T N_{r} \times 1}=\operatorname{vec}(\mathbf{W})$, $\mathbf{h}_{\chi} \in \mathbb{C}^{T N_{r} \times 1}=\operatorname{vec}(\mathbf{X H})$ and $\mathbf{h}_{u} \in \mathbb{C}^{T N_{r} \times 1}$ is a column vector that contains channel fade realizations corresponding to non-zero elements of $\mathbf{X}_{u}$, the transmission matrix of the $u$ th transmission group.

At the receiver side, assuming perfect channel state information (P-CSI), the equivalent signal model of (14) is considered and a maximum likelihood (ML) detector is used to achieve the optimum BER performance as

$$
\left(\hat{l}_{1}, \hat{l}_{2}, \ldots, \hat{l}_{N}\right)=\arg \min _{l_{1}, l_{2}, \ldots, l_{N}}\left\|\mathbf{y}-\sum_{u=1}^{N} \mathbf{h}_{u}\right\|^{2} .
$$

Then, the overall computational complexity of the ML detector (15) is evaluated in terms of real multiplications as $\sim \mathcal{O}\left(T N_{r} 2^{m T+1}\right)$, since each of $2^{m T}$ decision metric calculations requires $2 T N_{r}$ real multiplications for each $\|\cdot\|^{2}$ operation.

\section{PERFormance AND CAPACITy ANALYSES}

In this section, based on our system model of Section II, we present error performance and capacity analyses for the proposed ST-MBM scheme.

In the ST-MBM scheme, correlated and uncorrelated fading channels are considered and the correlated channel matrix $\mathbf{H}$ is modeled through the uncorrelated Rayleigh fading channel matrix $\tilde{\mathbf{H}} \in \mathbb{C}^{P \times N_{r}}$, whose elements are i.i.d. complex Gaussian random variables with distribution of $\mathcal{C N}(0,1)$, as

$$
\mathbf{H}=\mathbf{R}_{t}^{1 / 2} \tilde{\mathbf{H}} \mathbf{R}_{r}^{1 / 2} .
$$

where $\mathbf{R}_{t}$ and $\mathbf{R}_{r}$ denote transmit and receive correlation matrices with dimensions of $P \times P$ and $N_{r} \times N_{r}$, respectively.

In this study, the transmit correlation matrix $\mathbf{R}_{t}$ is determined by considering two different correlation models: the Kronecker model [34] and the equicorrelation model [14]. The Kronecker model is used for the correlation among the fades of different transmit antennas, while the equicorrelation model is considered for the correlation among the channel states of each transmit antenna. $\mathbf{R}_{t}$ is given in (17), where $r_{a}$ and $r_{b}$ are the correlation coefficients between the transmit antennas and channel states, respectively. On the other hand, the receive correlation matrix $\mathbf{R}_{r}$ is characterized by using the Kronecker model [34] and given as

$$
\mathbf{R}_{r}=\left[\begin{array}{ccccc}
1 & r_{a} & r_{a}^{2} & \cdots & r_{a}^{N_{r}-1} \\
r_{a} & 1 & r_{a} & \cdots & r_{a}^{N_{r}-2} \\
r_{a}^{2} & r_{a} & 1 & \cdots & r_{a}^{N_{r}-3} \\
\vdots & \vdots & \vdots & \ddots & \vdots \\
r_{a}^{N_{r}-1} & r_{a}^{N_{r}-2} & r_{a}^{N_{r}-3} & \cdots & 1
\end{array}\right]
$$

It is worth noting that $0<r_{a}, r_{b}<1$.

\section{A. Performance Analysis}

In this subsection, the theoretical ABEP performance of the ST-MBM scheme is analyzed. Considering a commonly used upper bounding technique [35], the ABEP of the system is given as

$$
P_{b} \leq \frac{1}{2^{\kappa}} \sum_{\mathbf{X}}\left[\frac{1}{\kappa} \sum_{\hat{\mathbf{X}}} \operatorname{Pr}(\mathbf{X} \rightarrow \hat{\mathbf{X}}) e(\mathbf{X}, \hat{\mathbf{X}})\right]
$$

where $\kappa$ is the number of incoming bits, i.e., $\kappa=m T$, $\operatorname{Pr}(\mathbf{X} \rightarrow \hat{\mathbf{X}})$ is the pairwise error probability (PEP) and $e(\mathbf{X}, \hat{\mathbf{X}})$ is the number of bit errors occurred for the corresponding pairwise error event.

In order to obtain the PEP of the ST-MBM scheme, first, conditional PEP (CPEP) is derived, which can be given in terms of the $Q$-function as follows

$$
P_{r}(\mathbf{X} \rightarrow \hat{\mathbf{X}} \mid \mathbf{H})=Q\left(\sqrt{\frac{\Lambda}{2 N_{0}}}\right)
$$

where $\Lambda$ is given, for $\varphi=1 / N_{0}$, as

$$
\Lambda=\|\varphi(\mathbf{X}-\hat{\mathbf{X}}) \mathbf{H}\|^{2}
$$

Then, for the following difference matrix

$$
\boldsymbol{\Delta}=(\mathbf{X}-\hat{\mathbf{X}})^{H}(\mathbf{X}-\hat{\mathbf{X}})
$$


and $\mathbf{R}_{s}=\mathbf{R}_{r} \otimes \mathbf{R}_{t}, \Lambda$ is rewritten in quadratic form as

$$
\begin{aligned}
\Lambda & =\|\varphi(\mathbf{X}-\hat{\mathbf{X}}) \mathbf{H}\|^{2} \\
& =\operatorname{Tr}\left(\mathbf{H}^{H}(\mathbf{X}-\hat{\mathbf{X}})^{H}(\mathbf{X}-\hat{\mathbf{X}}) \mathbf{H}\right) \\
& =\operatorname{vec}\left(\mathbf{H}^{H}\right)^{H}\left(\mathbf{I}_{N_{r}} \otimes \boldsymbol{\Delta}\right) \operatorname{vec}\left(\mathbf{H}^{H}\right) \\
& =\operatorname{vec}\left(\tilde{\mathbf{H}}^{H}\right)^{H}\left(\mathbf{R}_{s}{ }^{1 / 2}\right)^{H}\left(\mathbf{I}_{N_{r}} \otimes \boldsymbol{\Delta}\right) \mathbf{R}_{s}{ }^{1 / 2} \operatorname{vec}\left(\tilde{\mathbf{H}}^{H}\right) .
\end{aligned}
$$

Substituting (23) into the following alternative expression

$$
Q(x)=\frac{1}{\pi} \int_{0}^{\pi / 2} \exp \left(-\frac{x^{2}}{2 \sin ^{2} \theta}\right) d \theta
$$

and considering

$$
\boldsymbol{\Gamma}=\left(\mathbf{R}_{s}^{1 / 2}\right)^{H}\left(\mathbf{I}_{N_{r}} \otimes \boldsymbol{\Delta}\right) \mathbf{R}_{s}{ }^{1 / 2}
$$

the CPEP of the system (20) is given in a positive semidefinite quadratic form as

$$
\begin{aligned}
P_{r}(\mathbf{X} & \rightarrow \hat{\mathbf{X}} \mid \mathbf{H}) \\
& =\frac{1}{\pi} \int_{0}^{\pi / 2} \exp \left(-\varphi \frac{\operatorname{vec}\left(\tilde{\mathbf{H}}^{H}\right)^{H} \boldsymbol{\Gamma} \operatorname{vec}\left(\tilde{\mathbf{H}}^{H}\right)}{4 \sin ^{2} \theta}\right) d \theta
\end{aligned}
$$

Then, the PEP of the system is derived by averaging (26) over the channel matrix $\mathbf{H}$ through the moment generating function (MGF) approach as follows

$$
P_{r}(\mathbf{X} \rightarrow \hat{\mathbf{X}})=\frac{1}{\pi} \int_{0}^{\pi / 2} \mathcal{M}_{\Gamma}\left(-\frac{\varphi}{4 \sin ^{2} \theta}\right) d \theta
$$

The MGF of (27) is evaluated using the expression for the MGF of a quadratic form $\mathbf{z}^{H} \mathbf{Q z}$, involving the vector $\mathbf{z}$ and the Hermitian matrix $\mathbf{Q}$, given in [36] as

$$
\mathcal{M}(s)=\frac{\exp \left[s \overline{\mathbf{z}}^{H} \mathbf{Q}\left(\mathbf{I}-s \mathbf{K}_{\mathbf{z}} \mathbf{Q}\right)^{-1} \overline{\mathbf{z}}\right]}{\left(\mathbf{I}-s \mathbf{K}_{\mathbf{z}} \mathbf{Q}\right)}
$$

where $\overline{\mathbf{z}}$ is the mean vector and $\mathbf{K}_{\mathbf{z}}$ is the covariance matrix that is given as

$$
\mathbf{K}_{\mathbf{z}}=E\left\{\mathbf{z}^{H} \mathbf{z}\right\}-\overline{\mathbf{z}}^{H} \overline{\mathbf{z}} .
$$

Since $\operatorname{vec}\left(\tilde{\mathbf{H}}^{H}\right)$ is a complex Gaussian channel vector whose elements are distributed with zero mean and unit variance, for $\mathbf{z}=\operatorname{vec}\left(\tilde{\mathbf{H}}^{H}\right)$ and $\mathbf{Q}=\boldsymbol{\Gamma}$, the mean vector and the covariance matrix of $\operatorname{vec}\left(\tilde{\mathbf{H}}^{H}\right)$ respectively become $\overline{\mathbf{z}}=\overline{\operatorname{vec}\left(\tilde{\mathbf{H}}^{H}\right)}=0$ and $\mathbf{K}_{\mathbf{z}}=\mathbf{I}_{P N_{r}}$. Therefore, the exact PEP of the ST-MBM scheme is calculated from the following integral

$$
P_{r}(\mathbf{X} \rightarrow \hat{\mathbf{X}})=\frac{1}{\pi} \int_{0}^{\pi / 2} \operatorname{det}\left[\mathbf{I}_{P N_{r}}+\frac{\varphi}{4 \sin ^{2} \theta} \boldsymbol{\Gamma}\right]^{-1} d \theta .
$$

It is worth noting that (30) can be easily calculated by numerical integration. Also, a closed form upper-bound expression for (30) is obtained by letting $\theta=\frac{\pi}{2}$ as

$$
P_{r}(\mathbf{X} \rightarrow \hat{\mathbf{X}}) \leq \frac{1}{2} \operatorname{det}\left(\mathbf{I}_{P N_{r}}+\frac{\varphi}{4} \boldsymbol{\Gamma}\right)^{-1} .
$$

\section{B. Capacity Analysis}

The amount of information conveyed between the transmission vector $\mathbf{x}$ and the received vector $\mathbf{y}$ is defined as the mutual information and is given for the MIMO channel matrix $\mathbf{H}$ as

$$
I(\mathbf{y} ; \mathbf{x})=\mathbb{E}_{\mathbf{H}}\{H(\mathbf{y} \mid \mathbf{H})-H(\mathbf{y} \mid \mathbf{x}, \mathbf{H})\} .
$$

However, for the ST-MBM scheme, when the equivalent signal model (14) of the ST-MBM is considered, since incoming information bits modulate channel elements and carry no information with an ordinary modulation, the mutual information is defined as amount of information conveyed between the received signal $\mathbf{y}$ and the channel vector $\mathbf{h} \chi$ [37], and can be given by (33).

The conditional probability density function (p.d.f.) of the equivalent received signal vector of (14) is given by

$$
P(\mathbf{y} \mid \mathbf{h} \chi)=\frac{1}{\left(\pi N_{0}\right)^{T N_{r}}} \exp \left(-\frac{\|\mathbf{y}-\mathbf{h} \chi\|^{2}}{N_{0}}\right) .
$$

Since all possible $\mathbf{h} \chi$ are equiprobable, we have $P(\mathbf{h} \chi)=$ $1 / 2^{\kappa}$. Substituting (34) and $P(\mathbf{h} \chi)=1 / 2^{\kappa}$ into (33), the

$$
\begin{aligned}
I(\mathbf{y} ; \mathbf{h} \chi) & =H(\mathbf{y})-H(\mathbf{y} \mid \mathbf{h} \chi) \\
& =\frac{1}{T} \sum_{\mathbf{h} \chi} \int_{-\infty}^{\infty} \cdots \int_{-\infty}^{\infty} P(\mathbf{y} \mid \mathbf{h} \chi) P(\mathbf{h} \chi) \times \log _{2}\left(\frac{P(\mathbf{y} \mid \mathbf{h} \chi)}{\sum_{\hat{\mathbf{h}} \chi} P(\mathbf{y} \mid \hat{\mathbf{h}} \chi) P(\hat{\mathbf{h}} \chi)}\right) d \mathbf{y}
\end{aligned}
$$

$$
\begin{aligned}
& I(\mathbf{y} ; \mathbf{h} \chi) \\
= & \frac{1}{T}\left(\kappa-\left[\frac{1}{2^{\kappa}} \times \sum_{\mathbf{h} \chi} \frac{1}{\left(\pi N_{0}\right)^{T N_{r}}} \int_{\mathbf{y} \in \mathbb{C}^{T N_{r}}} \exp \left(-\frac{\|\mathbf{y}-\mathbf{h} \chi\|^{2}}{N_{0}}\right) \times \log _{2}\left(\sum_{\substack{\hat{\mathbf{h}} \chi \neq \mathbf{h} \chi \\
\mathbf{n}}} \exp \left(\frac{\|\mathbf{y}-\mathbf{h} \chi\|^{2}-\|\mathbf{y}-\hat{\mathbf{h}} \chi\|^{2}}{N_{0}}\right)\right) d \mathbf{y}\right]\right) \\
= & \frac{1}{T}\left(\kappa-\left[\frac{1}{2^{\kappa}} \times \sum_{\mathbf{h} \chi} \frac{1}{\left(\pi N_{0}\right)^{T N_{r}}} \int_{\mathbf{y} \in \mathbb{C}^{T N_{r}}} \exp \left(-\frac{\|\mathbf{y}-\mathbf{h} \chi\|^{2}}{N_{0}}\right) \times \log _{2}\left(1+\sum_{\substack{\hat{\mathbf{h}} \chi \\
\hat{\mathbf{h}} \chi \neq \mathbf{h} \chi}} \exp \left(\frac{\|\mathbf{y}-\mathbf{h} \chi\|^{2}-\|\mathbf{y}-\hat{\mathbf{h}} \chi\|^{2}}{N_{0}}\right)\right) d \mathbf{y}\right]\right)
\end{aligned}
$$




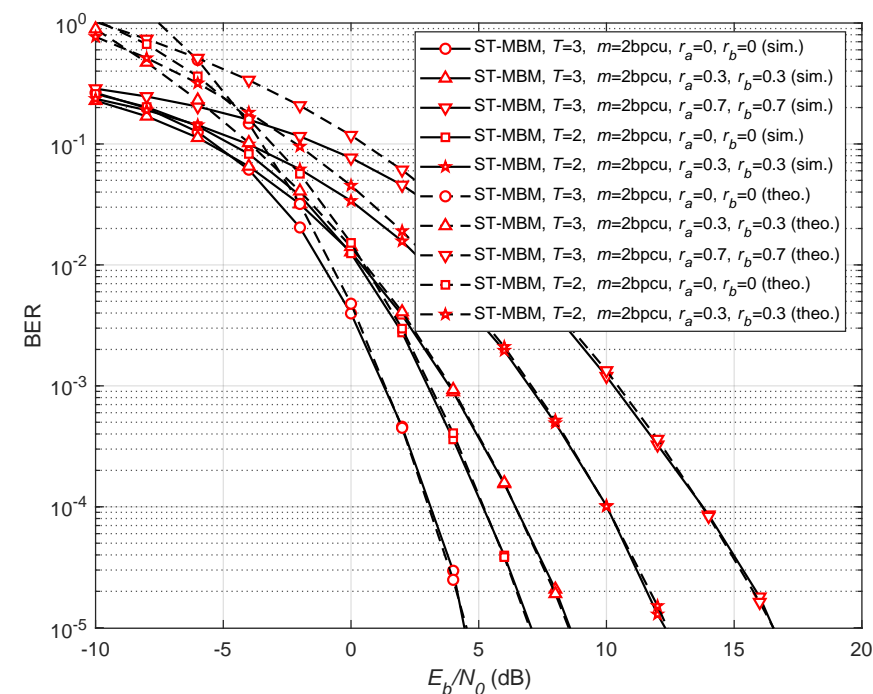

Fig. 2. Theoretical and simulation results of the ST-MBM scheme for $T=2$ and $T=3$ under different channel correlation values.

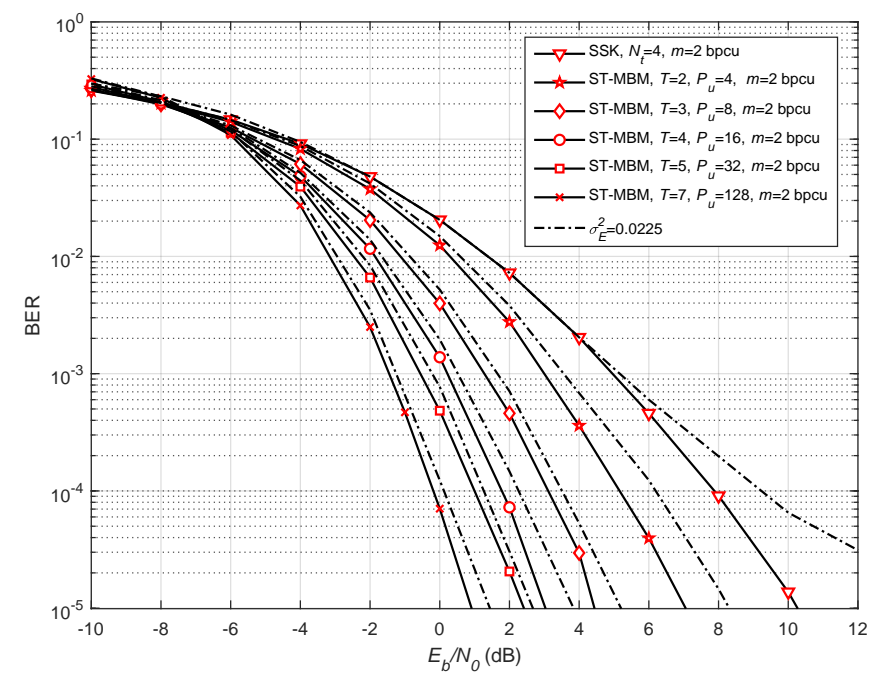

Fig. 3. BER performance of the SSK and ST-MBM schemes for $T \in$ $\{2,3,4,5,7\}$ and $m=2$ bpcu with perfect and imperfect channel estimation.

mutual information of the ST-MBM scheme using $T$ time slots, $I(\mathbf{y} ; \mathbf{h} \chi)$, results in (36), where the factor of $1 / T$ comes from $T$ channel uses. Then, using the Jensen's inequality and applying some algebraic manipulations [38], a lower bound is obtained for $I(\mathbf{y} ; \mathbf{h} \chi)$ as

$$
\begin{aligned}
& I(\mathbf{y} ; \mathbf{h} \chi) \geq \\
& \frac{1}{T}\left(\kappa-\left[1+\frac{1}{2^{\kappa}} \times \sum_{\mathbf{h} \chi} \sum_{\substack{\hat{\mathbf{h}} \chi \\
\mathbf{h} \chi \neq \mathbf{h} \chi}} \exp \left(\frac{-\|\mathbf{h} \chi-\hat{\mathbf{h}} \chi\|^{2}}{4 N_{0}}\right)\right]\right) .
\end{aligned}
$$

\section{Simulation Results}

In this section, BER performance of the ST-MBM scheme is investigated through comprehensive computer simulations.

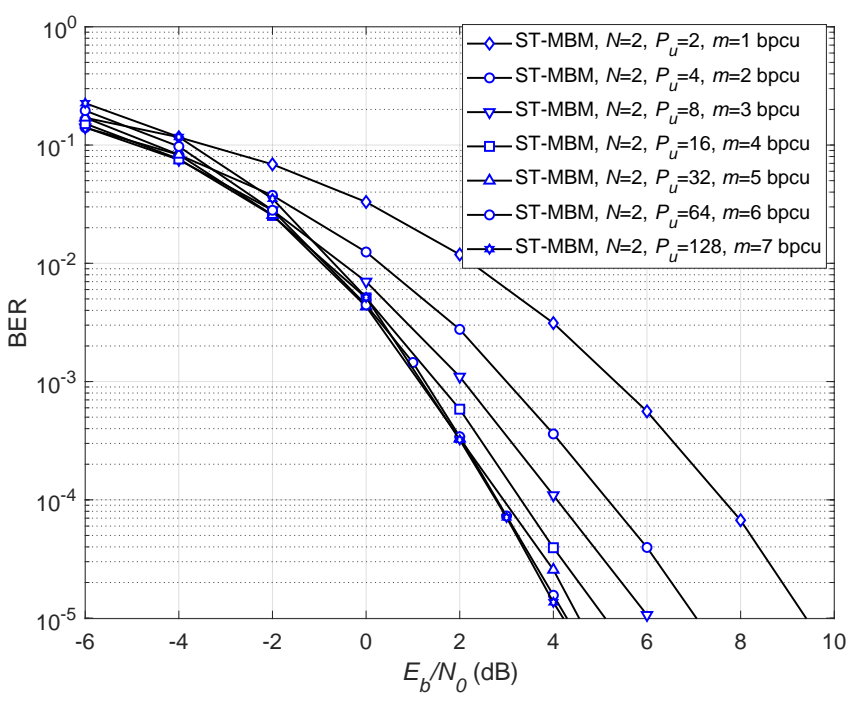

Fig. 4. BER performance of the ST-MBM scheme for $T=2$ and $m=$ $1,2,3,4,5,6,7$ bpcu.

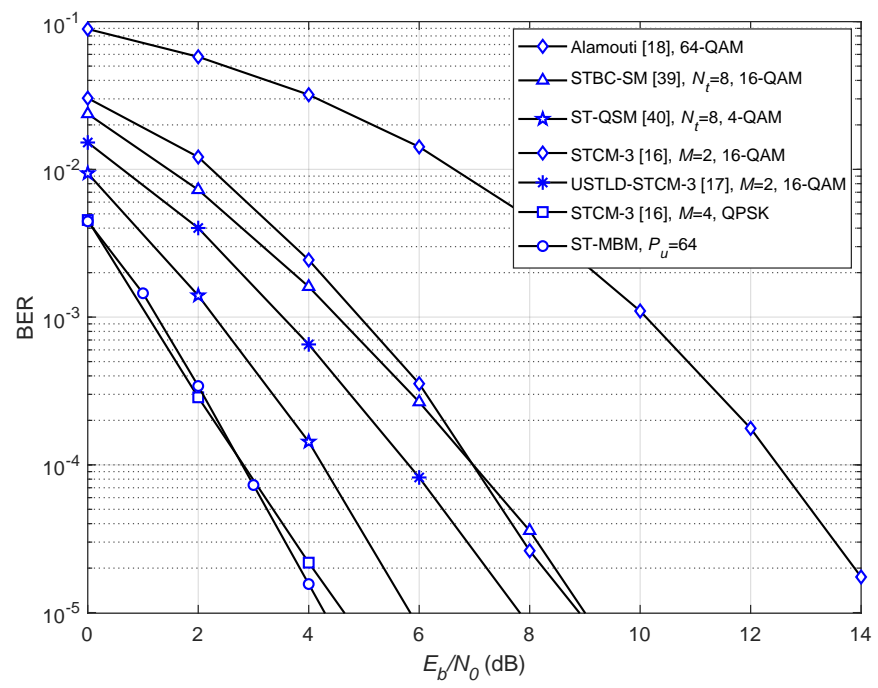

Fig. 5. BER performance of ST-MBM, STCM [16], USTLD-STCM [17], STBC-SM [39], ST-QSM [40], Alamouti's STBC [18] and SSK schemes for $m=6$ bpcu.

For different spectral efficiency values and transmit diversity gains, the BER performance and mutual information analysis of the ST-MBM scheme are evaluated and its superior error performance over existing systems are shown. All computer simulations are depicted as a function of the received energy per bit to noise ratio $\left(E_{b} / N_{0}\right)$ for $N_{r}=4$.

In Fig. 2, the theoretical ABEP performance of ST-MBM schemes designed for $T=2$ and $T=3$ is depicted for correlated and uncorrelated fading conditions. The results show that the theoretical ABEP results of the ST-MBM scheme are consistent with the computer simulation results at high $E_{b} / N_{0}$ values. Also, increasing correlation coefficients $r_{a}$ and $r_{b}$ apparently results in noticeable performance degradation and should be carefully monitored by the system designer.

To show the effect of different transmit diversity gains at a certain spectral efficiency value, in Fig. 3, the BER 


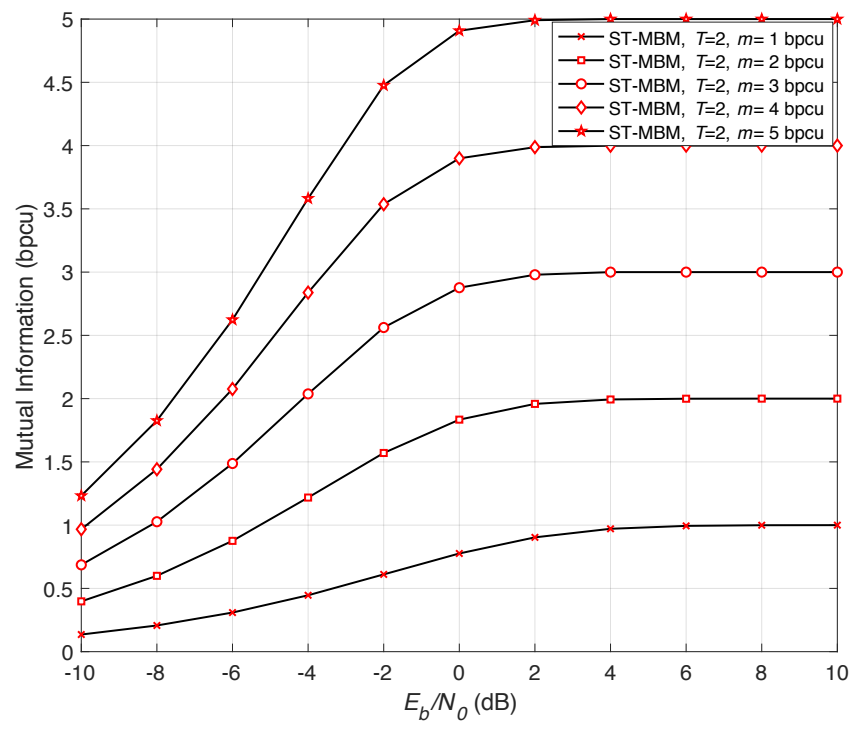

Fig. 6. Mutual information of the ST-MBM scheme with varying spectral efficiencies for $T=2$.

performance of the ST-MBM scheme achieving a transmit diversity order of $T$ with and without channel estimation errors, is provided. For each case, to achieve the same spectral efficiency value of $m=2 \mathrm{bpcu}$, a total of $P=2^{m T}$ channel fade realizations are considered for $N=2$ transmission groups, where $P=N P_{u}$. Then, to analyze the effect of imperfect channel state knowledge on the BER performance of the proposed ST-MBM scheme, the estimated channel matrix is assumed to be $\hat{\mathbf{H}}=\mathbf{H}+\mathbf{E}$, where $\mathbf{E}$ is the matrix of channel estimation errors whose entries are i.i.d. Gaussian random variables with the distribution of $\mathcal{C N}\left(0, \sigma_{E}^{2}\right)$. Thus, the entries of the overall estimated channel matrix $\hat{\mathbf{H}}$ are assumed to be distributed with $\mathcal{C N}\left(0,1+\sigma_{E}^{2}\right)$. The shown results clearly demonstrate the superior performance of the ST-MBM scheme with increasing transmit diversity gains, which is significantly beyond the performance of the SSK scheme with unity transmit diversity gain. Moreover, it can be deduced from Fig. 3 that the ST-MBM schemes achieving $T$ th order transmit diversity are more robust to channel state errors at high $E_{b} / N_{0}$ values compared to the traditional SSK scheme. We also observe from Fig. 3 that the proposed ST-MBM scheme would be suitable for future ultra-reliable communication systems that require high diversity gains.

In Fig. 4, BER performance of the ST-MBM scheme with increasing spectral efficiency values is investigated. It can be observed from this figure that unlike the traditional MIMO systems, as the spectral efficiency value increases, the error performance gradually improves. However, the system performance appears to be saturated at spectral efficiency values of 6 and $7 \mathrm{bpcu}$, which shows a similar behavior with the simulation results of the classical SSK/MBM schemes that saturate at high spectral efficiency values [16]. This phenomenon shows that as the ST-MBM scheme creates a virtual constellation that has randomly distributed elements while attaining transmit diversity gains, it retains the inherent maximum capacity achieving property of the classical SSK

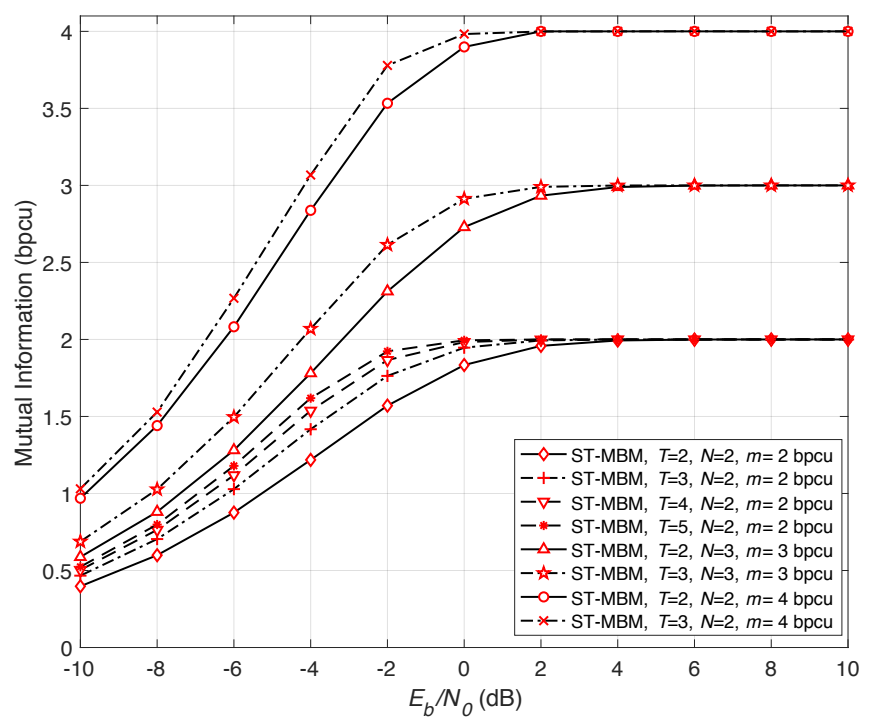

Fig. 7. Mutual information of the ST-MBM scheme with increasing transmit diversity orders for $m=2,3$ and $4 \mathrm{bpcu}$.

scheme.

In Fig. 5, for $m=6 \mathrm{bpcu}$, the error performance of the STMBM scheme is compared with the classical SSK scheme and the existing state-of-the-art STBC-based systems that achieve second order transmit diversity gain. The significant performance improvement of the ST-MBM scheme over common STBC-based systems including Alamouti's STBC [18], STBCSM [39] and space-time quadrature SM (ST-QSM) [40] is clearly observed. Furthermore, we compare the performance of the proposed scheme with recently developed STBCbased MBM systems including space-time channel modulation (STCM) [16] and USTLD-STCM [17] to achieve $m=6$ bpcu. In this comparison, $M=2 \mathrm{RF}$ mirrors are utilized with 16-QAM signal constellation, while in the ST-MBM scheme, $N=2$ transmission groups with $P_{u}=64$ channel fade realizations are used without ordinary modulation. Computer simulation results show that at a BER value of $10^{-5}$, the ST-MBM scheme exhibits 3.5 and $1.5 \mathrm{~dB}$ gains in required $E_{b} / N_{0}$ compared to STCM [16] and USTLD-STCM [17], respectively. However, for the same spectral efficiency, when STCM employs $M=4$ RF mirrors with QPSK modulation, its BER performance significantly improves, while it is still 0.5 $\mathrm{dB}$ behind the ST-MBM scheme at the BER value of $10^{-5}$. Compared to STCM, the proposed ST-MBM scheme achieves almost $80 \%$ reduction in transceiver complexity, where the decoding complexity of STCM with two RF chains as well as in-phase and quadrature $(\mathrm{I} / \mathrm{Q})$ modulation, in terms of real multiplications, is $\sim \mathcal{O}\left(10 T N_{r} 2^{m T}\right)$ while the corresponding value is $\sim \mathcal{O}\left(T N_{r} 2^{m T+1}\right)$ for the ST-MBM scheme. In conclusion, we note that the proposed ST-MBM scheme provides a remarkable reduction in both transmitter and receiver complexity by eliminating the need for multiple RF chains and I/Q modulation and enabling a significant reduction in the number of operations required for ML detection.

In Figs. 6 and 7, the mutual information curves of the STMBM scheme that achieves a $T$ th order transmit diversity 
gain, is depicted for different spectral efficiency values. Fig. 6 shows that as $E_{b} / N_{0}$ increases, each curve converges to its attainable mutual information value. Additionally, in Fig. 7, for spectral efficiency values of $m=2,3$ and $4 \mathrm{bpcu}$, the mutual information results of the ST-MBM scheme for different transmit diversity orders are compared. The mutual information results of the ST-MBM scheme are compared at $m=2 \mathrm{bpcu}$, when $T$ varies from two to five, while at $m=3$ and $4 \mathrm{bpcu}$, transmit diversity orders of three and four are considered. Although the mutual information of the ST-MBM scheme is scaled with the transmit diversity order of $T$ due to the number of channel uses (37), the results reveal that as the transmit diversity order, $T$, increases the system converges faster to its achievable mutual information.

\section{Conclusion}

In this paper, we have presented a general framework for space-time coded IM systems and introduced the ST-MBM scheme as the first STBC-based transmission scheme that uses a single RF chain at the transmitter while achieving various transmit diversity gains through MBM and time dispersion. Theoretical error performance analysis of the STMBM scheme for correlated and uncorrelated channel fadings has been investigated. Additionally, a lower bound has been derived for the mutual information of the ST-MBM scheme. Furthermore, through extensive computer simulations, the superior error performance of the proposed ST-MBM scheme with significantly lower decoding complexity over existing STBC-based transmission schemes has been demonstrated. The flexibility to achieve higher spectral efficiencies and various transmit diversity gains makes the ST-MBM scheme highly suitable for beyond 5G and ultra-reliable lowlatency communications (URLLC) applications. Our future work will focus on the enhancement of the proposed STMBM scheme through the use of multiple RF chains and/or ordinary modulations, low-complexity detection algorithms as well as performance analysis of the proposed system over poorly scattered millimeter wave (mmWave) channels.

\section{REFERENCES}

[1] G. J. Foschini, "Layered space-time architecture for wireless communication in a fading environment when using multi-element antennas," Bell Lab. Tech. J., vol. 1, no. 2, pp. 41-59, Autumn, 1996.

[2] E. Basar, "Index modulation techniques for $5 \mathrm{G}$ wireless networks," IEEE Commun. Mag., vol. 54, no. 7, pp. 168-175, July 2016.

[3] R. Mesleh, H. Haas, S. Sinanovic, C. W. Ahn, and S. Yun, "Spatial modulation," IEEE Trans. Veh. Technol., vol. 57, no. 4, pp. 22-28, July 2008.

[4] J. Jeganathan, A. Ghrayeb, L. Szczecinski, and A. Ceron, "Space shift keying modulation for MIMO channels," IEEE Trans. Wireless Commun., vol. 8, no. 7, pp. 3692-3703, July 2009.

[5] E. Basar, M. Wen, R. Mesleh, M. Di Renzo, Y. Xiao, and H. Haas, "Index modulation techniques for next-generation wireless networks," IEEE Access, vol. 5, pp. 16693-16746, Aug. 2017.

[6] E. Basar, U. Aygolu, E. Panayirci, and H. V. Poor, "Orthogonal frequency division multiplexing with index modulation," IEEE Trans. Signal Process., vol. 61, no. 22, pp. 5536-5549, Nov. 2013.

[7] E. Basar, "On multiple-input multiple-output OFDM with index modulation for next generation wireless networks," IEEE Trans. Signal Process., vol. 64, no. 15, Aug. 2016.

[8] G. Kaddoum, M. F. Ahmed, and Y. Nijsure, "Code index modulation: A high data rate and energy efficient communication system," IEEE Commun. Lett., vol. 19, no. 2, pp. 175-178, Feb. 2015.
[9] Q. Li, M. Wen, E. Basar, and F. Chen, "Index modulated OFDM spread spectrum," IEEE Trans. Wireless Commun., vol. 17, no. 4, pp. 23602374, Apr. 2018.

[10] R. Mesleh, H. Elgala, and H. Haas, "Optical spatial modulation," IEEE/OSA J. Optical Commun. and Netw., vol. 3, no. 3, pp. 234-244, Mar. 2011.

[11] A. Yesilkaya, E. Basar, F. Miramirkhani, E. Panayirci, M. Uysal, and H. Haas, "Optical MIMO-OFDM with generalized LED index modulation," IEEE Trans. Commun., vol. 65, no. 8, pp. 3429-3441, Aug. 2017.

[12] A. K. Khandani, "Media-based modulation: A new approach to wireless transmission," in Proc. IEEE Int. Symp. Inf. Theory (ISIT), July 2013, pp. 3050-3054.

[13] R. Mesleh, S. S. Ikki, and H. M. Aggoune, "Quadrature spatial modulation," IEEE Trans. Veh. Technol., vol. 64, no. 6, pp. 2738-2742, June 2015.

[14] Y. Naresh and A. Chockalingam, "On media-based modulation using RF mirrors," IEEE Trans. Veh. Technol., vol. 66, no. 6, pp. 4967-4983, June 2017.

[15] I. Yildirim, E. Basar, and I. Altunbas, "Quadrature channel modulation," IEEE Wireless Commun. Lett., vol. 6, no. 6, pp. 790-793, Dec. 2017.

[16] E. Basar and I. Altunbas, "Space-time channel modulation," IEEE Trans. Veh. Technol., vol. 66, no. 8, pp. 7609-7614, Feb. 2017.

[17] N. Pillay and $\mathrm{H}$. $\mathrm{Xu}$, "Uncoded space-time labeling diversity-application of media-based modulation with RF mirrors," IEEE Commun. Lett., vol. 22, no. 2, pp. 272-275, Feb. 2018.

[18] S. M. Alamouti, "A simple transmit diversity technique for wireless communications," IEEE J. Sel. Areas Commun., vol. 16, no. 8, pp. 14511458, Oct. 1998.

[19] H. Xu, K. Govindasamy, and N. Pillay, "Uncoded space-time labeling diversity," IEEE Commun. Lett., vol. 20, no. 8, pp. 1511-1514, Aug. 2016.

[20] M. Yüzgeçcioğlu and E. Jorswieck, "Performance of media-based modulation in multi-user networks," Proc. the Int. Symp. Wireless Commun. Systems, Aug. 2017.

[21] B. Shamasundar and A. Chockalingam, "Multiuser media-based modulation for massive MIMO systems," in Proc. Signal Process. Advances in Wireless Commun. (SPAWC), IEEE 18th Int. Workshop, Dec. 2017.

[22] B. Shamasundar, S. Jacob, L. N. Theagarajan, and A. Chockalingam, "Media-based modulation for the uplink in massive MIMO systems," IEEE Trans. Veh. Technol., vol. 67, no. 9, pp. 8169 - 8183, Sept. 2018.

[23] L. Zhang, M. Zhao, and L. Li, "Low-complexity multi-user detection for MBM in uplink large-scale MIMO systems," IEEE Commun. Lett., vol. 22, pp. 1568 - 1571, Apr. 2018.

[24] Y. Naresh and A. Chockalingam, "A low-complexity maximumlikelihood detector for differential media-based modulation," IEEE Commun. Lett., vol. 21, no. 10, pp. 2158-2161, Mar. 2017.

[25] S. Jacob, A. Chockalingam et al., "Detection of generalized media-based modulation signals using multi-layered message passing," in Proc. 2018 IEEE 87th Veh. Technol. Conf.(VTC Spring), June 2018.

[26] Y. Naresh and A. Chockalingam, "Performance analysis of media-based modulation with imperfect channel state information," IEEE Trans. Veh. Technol., vol. 67, pp. 4192 - 4207, May 2018.

[27] — "Full-duplex media-based modulation," in Proc. IEEE Globecom Workshops (GC Wkshps), Dec. 2017, pp. 1-6.

[28] I. Yildirim, E. Basar, and G. Kurt, "Media-based modulation for secrecy communications," IET Electron. Lett., vol. 54, pp. 789-791, June 2018.

[29] L. Zhang and M. Zhao, "Secrecy enhancement for media-based modulation via probabilistic optimization," IEEE Commun. Lett., Dec. 2018.

[30] E. Basar, "Media-based modulation for future wireless systems: A tutorial," arXiv preprint arXiv:1811.08730, Nov. 2018.

[31] C. E. Shannon, "A mathematical theory of communication," $A C M$ SIGMOBILE Mobile Computing and Commun. Review, vol. 5, no. 1, pp. 3-55, 2001.

[32] H. Jafarkhani, Space-time coding: Theory and practice. Cambridge University Press, 2005.

[33] R. Mesleh, O. Hiari, A. Younis, and S. Alouneh, "Transmitter design and hardware considerations for different space modulation techniques," IEEE Trans. Wireless Commun., vol. 16, no. 11, pp. 7512-7522, Nov. 2017.

[34] A. Paulraj, R. Nabar, and D. Gore, Introduction to space-time wireless communications. Cambridge University Press, 2003.

[35] M. K. Simon and M.-S. Alouini, Digital communication over fading channels. John Wiley \& Sons, 2005. 
[36] G. L. Turin, "The characteristic function of Hermitian quadratic forms in complex normal variables," Biometrika, vol. 47, no. 1/2, pp. 199-201, June 1960.

[37] S. Sugiura, S. Chen, and L. Hanzo, "Space-time shift keying: A unified MIMO architecture," in Proc. IEEE Global Telecommun. Conf. (GLOBECOM), Dec. 2010.

[38] S. P. Herath, N. H. Tran, and T. Le-Ngoc, "Rotated multi-D constellations in Rayleigh fading: Mutual information improvement and pragmatic approach for near-capacity performance in high-rate regions," IEEE Trans. Commun., vol. 60, no. 12, pp. 3694-3704, Sep. 2012.

[39] E. Basar, U. Aygolu, E. Panayirci, and H. V. Poor, "Space-time block coded spatial modulation," IEEE Trans. Commun., vol. 59, no. 3, pp. 823-832, Dec. 2011.

[40] Z. Yigit and E. Basar, "Space-time quadrature spatial modulation," in Proc. IEEE 5th Int. Black Sea Conf. Commun. Netw.(BlackSeaCom), June 2017.

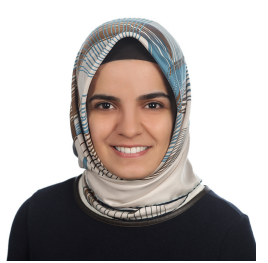

Zehra Yigit received her B.Sc. degree from University of Gaziantep, Turkey, in 2013, and her M. Sc. Degree from Istanbul Technical University, Turkey, in 2017. Currently, she is a Ph. D. student and research assistant at Istanbul Technical University, Electronics and Communication Engineering Department. She has served as a reviewer for IEEE, IET and Physical Communication (Elsevier) journals. Her research interests include MIMO systems, index modulation, space-time coding and cooperative communications.

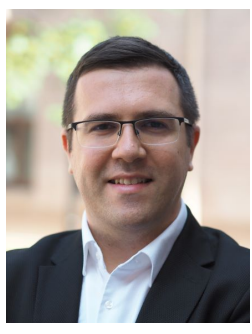

Ertugrul Basar (S'09-M'13-SM'16) received the B.S. degree (Hons.) from Istanbul University, Turkey, in 2007, and the M.S. and Ph.D. degrees from Istanbul Technical University, Turkey, in 2009 and 2013, respectively. He is currently an Associate Professor with the Department of Electrical and Electronics Engineering, Koç University, Istanbul, Turkey and the director of Communications Research and Innovation Laboratory (CoreLab). His primary research interests include MIMO systems, index modulation, waveform design, visible light communications, and signal processing for communications.

Recent recognition of his research includes the Science Academy (Turkey) Young Scientists (BAGEP) Award in 2018, Mustafa Parlar Foundation Research Encouragement Award in 2018, Turkish Academy of Sciences Outstanding Young Scientist (TUBA-GEBIP) Award in 2017, and the firstever IEEE Turkey Research Encouragement Award in 2017.

Dr. Basar currently serves as an Editor of the IEEE TRANSACTIONS ON COMmunications and Physical Communication (Elsevier), and as an Associate Editor of the IEEE COMMUNICATIONS LETTERS. He served as an Associate Editor for the IEEE ACCESS from 2016 to 2018. 